\title{
ON THE SYMMETRIC SQUARE: DEFINITIONS AND LEMMAS
}

\author{
YUVAL Z. FLICKER
}

\begin{abstract}
We define the symmetric square lifting for admissible and automorphic representations, from the group $H=H_{0}=\mathrm{SL}(2)$, to the group $G=\operatorname{PGL}(3)$, and derive its basic properties. This lifting is defined by means of Shintani character relations. The definition is suggested by the computation of orbital integrals (stable and unstable) in our On the symmetric square: Orbital integrals, Math. Ann. 279 (1987), 173-193. It is compatible with dual group homomorphisms $\lambda_{0}: \widehat{H} \rightarrow \widehat{G}$ and $\lambda_{1}: \widehat{H}_{1} \rightarrow \widehat{G}$, where $H_{1}=\operatorname{PGL}(2)$. The lifting is proven for induced, trivial and special representations, and both spherical functions and orthogonality relations of characters are studied.
\end{abstract}

\section{INTRODUCTION}

The purpose of this paper is to define the symmetric square lifting in terms of character relations, and derive basic properties of this lifting. This work is required for our study of this lifting of automorphic forms of $H(\mathbf{A})$ to $G(\mathbf{A})$, where $H=H_{0}=\mathrm{SL}(2)$ and $G=\operatorname{PGL}(3)$, by means of the trace formula.

The lifting is suggested by the symmetric square, or adjoint representation $\lambda_{0}: \widehat{H} \rightarrow \widehat{G}$ of the dual group $\widehat{H}=\operatorname{PGL}(2, \mathbf{C})$ of $H$ in $\widehat{G}=\operatorname{SL}(3, \mathbf{C})$. Put

$$
\sigma(g)=\mathscr{J}^{t} g^{-1} \mathcal{J}, \quad \mathscr{J}=\left(\begin{array}{lll}
0 & & 1 \\
& -1 & \\
1 & & 0
\end{array}\right) .
$$

Then $H$ is a $\sigma$-endoscopic group of $G$. But $G$ has another $\sigma$-endoscopic group, which is $H_{1}=\operatorname{PGL}(2)$. We write $\lambda_{1}: \widehat{H}_{1}=\operatorname{SL}(2, \mathbf{C}) \rightarrow \widehat{G}, h \mapsto\left(\begin{array}{ll}h & 0 \\ 0 & 1\end{array}\right)$. Via the Satake isomorphism, the maps $\lambda_{i}$ formally define the lifting $\pi=\lambda_{i}\left(\pi_{i}\right)$ of unramified $H_{i}(F)$-modules $\pi_{i}$ to unramified $G(F)$-modules $\pi$. Moreover, we introduce in $\S 1$ the dual maps $\lambda_{i}^{*}: \mathbf{H} \rightarrow \mathbf{H}_{i}$ from the Hecke algebra $\mathbf{H}$ of $G(F)$ to the Hecke algebra $\mathbf{H}_{i}$ of $H_{i}(F)$. It follows from the definitions that if $f_{i}=\lambda_{i}^{*}(f)$ then the spherical functions $f$ and $f_{i}$ have matching orbital integrals on the split torus.

In $\S 2$ we define lifting, denoted $\pi_{i}=\lambda_{i}(\pi)$, of admissible representations $\pi_{i}$ of $H_{i}(F)$ to such representations $\pi$ of $G(F)$, by means of character relations. The definition is suggested by the study [I, 33$]$, of orbital integrals. It generalizes the spherical case, and uses packets rather than a single irreducible. Basic

Received by the editors June 1, 1988 and, in revised form, January 11, 1990.

1980 Mathematics Subject Classification (1985 Revision). Primary 10D40, 10D30; Secondary 12A67, 12A85, 14G10, 22E55.

Partially supported by an NSF grant. 
examples of the stable lifting $\lambda_{0}$ are given. These concern induced, trivial, and special representations.

Section 3 concerns orthogonality relations for characters which are needed in the study [IV] of the local lifting. The cases of supercuspidal $G(F)$-modules and Steinberg $\pi$ are standard but useful. We also record without proof the twisted orthogonality relation for two tempered $G(F)$-modules which are not relevant. The proof follows closely that of the nontwisted case by Kazhdan [K]. It depends on the twisted analogue of the crucial appendix of $[\mathrm{K}]$; this is proven in [F] for a general group, and in [IV, (1.8)], in our case.

\section{Hecke Algebra}

1.1. Dual-groups. Let $F$ be a global or local field of characteristic zero. Put $G=\operatorname{PGL}(3), H=H_{0}=\mathrm{SL}(2)$, and $H_{1}=\operatorname{PGL}(2)=\mathrm{SO}(3)$. For any field $K$ denote by $G(K), H(K)$ and $H_{1}(F)$ the group of $K$-rational points of $G, H$ and $H_{1}$. Fix a separable algebraic closure $\bar{F}$ of $F$.

Let $\hat{G}=\operatorname{SL}(3, C)$ be the dual group of $G$ (for any reductive group $G$ the dual group $\widehat{G}$ is defined in [B], where it is denoted by $\left.{ }^{L} G\right)$. Consider the semidirect product $\widehat{G}^{\prime}=\widehat{G} \rtimes\langle\sigma\rangle ;\langle\sigma\rangle$ denotes the group generated by the automorphism

$$
\sigma(g)=\mathscr{J}^{t} g^{-1} \mathcal{J}, \quad \mathcal{J}=\left(\begin{array}{lll}
0 & & 1 \\
& -1 & \\
1 & & 0
\end{array}\right)
$$

of $G$ of order 2 .

The dual group $\widehat{H}$ of $H$ is $\operatorname{PGL}(2, \mathrm{C}) \simeq \mathrm{SO}(3, \mathrm{C})$; it is isomorphic to the centralizer of $1 \times \sigma$ in the connected component of 1 in $\widehat{G}^{\prime}$, and to the $\sigma$-centralizer $\widehat{G}_{1}^{\sigma}=\left\{g\right.$ in $\left.\widehat{G} ; g^{-1} \sigma(g)=1\right\}$ of 1 in $\widehat{G}$. The isomorphism is given by

$$
\left(\begin{array}{ll}
a & b \\
c & d
\end{array}\right) \rightarrow \frac{1}{x}\left(\begin{array}{ccc}
a^{2} & a b \sqrt{2} & b^{2} \\
a c \sqrt{2} & a d+b c & b d \sqrt{2} \\
c^{2} & c d \sqrt{2} & d^{2}
\end{array}\right) \quad(x=a d-b c)
$$

This map will be denoted by $\lambda$, or $\lambda_{0}: \widehat{H} \rightarrow \widehat{G}$.

The dual-group $\widehat{G}_{1}$ of $H_{1}$ is $\operatorname{SL}(2, \mathrm{C})$, and the map

$$
\lambda_{1}:\left(\begin{array}{ll}
a & b \\
c & d
\end{array}\right) \rightarrow\left(\begin{array}{lll}
a & 0 & b \\
0 & 1 & 0 \\
c & 0 & d
\end{array}\right)
$$

embeds $\widehat{H}_{1}$ in $\widehat{G}$. The image is the centralizer of $e \times \sigma$ in $\widehat{G}$, where $e$ is the diagonal matrix $(-1,1,1)$. Equivalently, it is the $\sigma$-centralizer $\widehat{G}_{e}^{\sigma}$ of $e$ in $\widehat{G}$.

1.2. Hecke algebra. Let $F$ be a $p$-adic field, $R=\{x$ in $F ;|x| \leq 1\}$ its ring of integers, $K=G(R)$ the standard maximal compact subgroup of $G(F)$, $\mathbf{H}=\mathbf{H}_{G}$ the convolution algebra of complex-valued compactly supported $K$ bi-invariant functions $f$ on $G(F)$. Let $\pi$ be an admissible irreducible representation of $G(F)$ on a space $V$. Put ${ }^{\sigma} \pi(g)=\pi(\sigma g)(g$ in $G(F))$. Then ${ }^{\sigma} \pi$ is an admissible irreducible representation of $G(F)$ on $V$. We say that $\pi$ is 
$\sigma$-invariant if $\pi$ is equivalent to ${ }^{\sigma} \pi$. In this case there is an invertible operator $A: V \rightarrow V$ with $\pi(\sigma g)=A \pi(g) A^{-1}(g$ in $G(F))$. Since $\pi$ is irreducible and $A^{2}$ intertwines $\pi$ with itself, $A^{2}$ is a scalar which we normalize to be 1 . We put $\pi(\sigma)=A$, and define the operator $\pi(f \times \sigma)=\pi(f) \pi(\sigma)$ to be the map which assigns $\int f(g) \pi(g) A v d g$ to $v$.

If $f$ is spherical (in $\left.\mathbf{H}_{G}\right)$ and $\pi(f \times \sigma) \neq 0$, then the image of $\pi(f \times \sigma)$ is one-dimensional and $\pi(k) \quad(k \in K)$ is the identity on the image of $\pi(f \times \sigma)$. Hence $\pi$ is unramified, and it lies in a representation $I$ of $G(F)$ induced from an unramified character $\eta$ of the upper triangular Borel subgroup $B(F)$, $\pi$ being the unique unramified constituent in the composition series of $I$. Fix $v$ in $V$ so that $w=\pi(f \times \sigma) v$ is nonzero. It is clear that $A w$ is also a $K$ fixed vector, and $A w \neq 0$, since $A(A w)=w \neq 0$. Hence, there is a constant $c$ with $A w=c w$. As $A^{2}=1, c$ is 1 or -1 . We replace $A$ by $c A$ to have $A w=w$. This normalization is compatible with the global normalization in terms of Whittaker models; see [IV, (1.1.1)].

The character $\eta$ is given by

$$
\eta((a, b, c))=\mu_{1}(a) \mu_{2}(b) \mu_{3}(c)
$$

on an element $\gamma=(a, b, c)$ in the diagonal torus $T$ of $G$, where $\mu_{i}$ are characters of $F^{\times}$with $\mu_{1} \mu_{2} \mu_{3}=1$. Let $\pi$ be a local uniformizer in $F$. Consider the element $t=\left(\mu_{1}(\boldsymbol{\pi}), \mu_{2}(\boldsymbol{\pi}), \mu_{3}(\boldsymbol{\pi})\right)$ in the diagonal torus $\widehat{T}$ of $\widehat{G}$. Then the equivalence class of the unramified representation $\pi$ is uniquely determined by the conjugacy class in $\widehat{G}$ of $t$.

The normalized orbital integral

$$
F_{f}(\gamma)=\Delta(\gamma) \int f\left(g^{-1} \gamma g\right) \frac{d g}{d t} \quad(g \text { in } T(F) \backslash G(F)),
$$

where

$$
\Delta(\gamma)=\left|\frac{a-b}{a} \frac{b-c}{b} \frac{a-c}{c}\right|,
$$

depends only on the image of $\gamma=(a, b, c)$ in $T(F) / T(R) \simeq X_{*}(T)$ when $f$ is spherical, and hence will be denoted by $F_{f}(\mathbf{n})$, $\mathbf{n}$ being the image of $\gamma$ in $X_{*}(T) \simeq\left\{\left(n_{1}, n_{2}, n_{3}\right) ; n_{i}\right.$ in $\left.\mathbf{Z}\right\} /\{(n, n, n) ; n$ in $\mathbf{Z}\}$. Implicit is a choice of Haar measures $d g, d t$ on $G(F)$ and $T(F)$. For $t=\left(t_{1}, t_{2}, t_{3}\right)$ in $\widehat{T}$ and $\mathbf{n}=\left(n_{1}, n_{2}, n_{3}\right)$ in $X_{*}(T)$, we put

$$
\mathbf{n}(t)=t_{1}^{n_{1}} t_{2}^{n_{2}} t_{3}^{n_{3}}
$$

The Satake transform $\check{f}$ of $f$ is defined by

$$
\check{f}(t)=|T(R)| \sum_{\mathbf{n}} F_{f}(\mathbf{n}) \mathbf{n}(t) \quad\left(\mathbf{n} \text { in } X^{*}(\widehat{T}) \simeq X_{*}(T)\right),
$$

where $|T(R)|$ denotes the volume of $T(R)=T(F) \cap K$ with respect to $d t$. The map $f \rightarrow \check{f}$ is an isomorphism from the algebra $\mathbf{H}_{G}$ to the algebra $\mathbf{C}[\widehat{T}]^{W}$ of finite Laurent series in $t \in \widehat{T}$ which are invariant under the action of the Weyl group $W$ of $\widehat{T}$ in $\widehat{G}$.

1.3. Lemma. Suppose that $\pi$ is unramified and $t=t(\eta)=t(\pi)$ is a corresponding element in $\widehat{T}$. If ${ }^{\sigma} \eta=\eta$, then for any $f$ in $\mathbf{H}_{G}$ we have

$$
\operatorname{tr} \pi(f \times \sigma)=\check{f}(t) .
$$


Proof. This is standard, hence omitted.

1.4. Definition. For $\delta$ in $G(F)$ put $\Phi_{f}^{\sigma}(\delta)=\int_{G_{\delta}^{o}(F) \backslash G(F)} f\left(g^{-1} \delta \sigma(g)\right) d g$, where $G_{\delta}^{\sigma}(F)$ is the $\sigma$-centralizer of $\delta$ in $G(F)$ (see [I, §3]). Also put $F_{f}^{\sigma}(\delta)=$ $\Delta_{0}(N \delta) \Phi_{f}^{\sigma}(\delta)$, where $N \delta$ is the norm of $\delta$ in $\operatorname{SL}(2, F)$ (see [I, $\left.\S 1\right]$, and (1.8) below). Here $\Delta_{0}$ is the usual $\Delta$-factor on $H(F)$. Finally, $T^{1-\sigma}(F)=\left\{t \sigma(t)^{-1}\right.$; $t$ in $T(F)\}$.

Lemma. We have

$$
\operatorname{tr} I(\eta, f \times \sigma)=\int_{T^{1-\sigma}(F) \backslash T(F)} \frac{1}{2}(\eta(\delta)+\eta(\tilde{\delta})) F_{f}^{\sigma}(\delta) d \delta .
$$

Proof. This is also standard, hence omitted.

1.5. Cases of $H$ and $H_{1}$. Considerations analogous to (1.2), (1.3) apply in the cases of the groups $H=H_{0}=\mathrm{SL}(2)$ and $H_{1}=\operatorname{PGL}(2) \simeq \mathrm{SO}(3)$, with respect to the maximal compact subgroups $K_{i}=H_{i}(R)$. Unramified representations $\pi_{0}, \pi_{1}$ are associated with $I_{0}\left(\mu_{1}, \mu_{2}\right), I_{1}\left(\mu, \mu^{-1}\right)$ and classes represented by $t_{0}=\left(z_{1}, z_{2}\right), t_{1}=\left(z, z^{-1}\right)$ in $\widehat{H}_{0}, \widehat{H}_{1}$. Here $z_{i}=\mu_{i}(\boldsymbol{\pi}), z=\mu(\boldsymbol{\pi})$. For $f_{i}$ in the Hecke algebras $H_{i}$ of compactly supported $K_{i}$-bi-invariant functions on $H_{i}(F)$, the Satake transform is

$$
\begin{aligned}
& \check{f}_{0}\left(\left(z_{1}, z_{2}\right)\right)=\left|T_{0}(R)\right| \sum_{n} F_{f_{0}}(n)\left(z_{1} / z_{2}\right)^{n}, \\
& \check{f}_{1}\left(\left(z, z^{-1}\right)\right)=\left|T_{1}(R)\right| \sum_{n} F_{f_{1}}(n) z^{n} .
\end{aligned}
$$

$\left|T_{i}(R)\right|$ denotes the volume of $T_{i}(R)=T_{i}(F) \cap K_{i}$ with respect to $d a_{i} . F_{f_{i}}(n)$ denotes the normalized orbital integral of $f_{i}$ at regular elements $(a, b)$ in $T_{i}(F)$ (diagonal subgroup of $\left.H_{i}(F)\right)$ with valuations $(n,-n),(i=0)$ and $\left(m_{1}, m_{2}\right), m_{1}-m_{2}=n(i=1)$. It depends on the choice of Haar measures $d h_{i}, d a_{i}$ on $H_{i}(F), T_{i}(F)$; but $\check{f}_{i}$ depends only on $d h_{i}$.

The standard computation alluded to in (1.3) shows that for spherical $f_{i}$, $\pi_{i}$, we have

$$
\operatorname{tr} \pi_{i}\left(f_{i}\right)=\check{f}_{i}\left(t_{i}\right) \quad\left(t_{i}=t_{i}\left(\pi_{i}\right)\right) .
$$

1.6. Unramified lifting. Recall (1.1) that we have maps $\lambda_{i}: \widehat{H}_{i} \rightarrow \widehat{G}$ and ((1.2), (1.5)) classes $t_{i}, t$ in $\widehat{H}_{i}, \widehat{G}$ for unramified representations $\pi_{i}, \pi$ of $H_{i}(F)$, $H(F) \quad(i=0,1)$.

Definition. The unramified representation $\pi_{i}$ lifts to $\pi$ through $\lambda_{i}$ if $t=$ $\lambda_{i}\left(t_{i}\right)$. In this case we write $\pi=\lambda_{i}\left(\pi_{i}\right)$.

The maps $\tilde{\lambda}_{i}^{*}: \mathbf{H} \rightarrow \mathbf{H}_{i}$ dual to $\lambda_{i}$ are defined by $f_{i}=\tilde{\lambda}_{i}^{*}(f)$ if $\check{f}_{i}\left(t_{i}\right)=$ $\check{f}\left(\lambda_{i}\left(t_{i}\right)\right)$ for all $t_{i}$ in $\widehat{T}_{i}$. Equivalently, $f_{i}=\tilde{\lambda}_{i}^{*}(f)$ if $\operatorname{tr} \pi_{i}(f)=\operatorname{tr} \pi(f)$ for all $\pi_{i}$ and $\pi=\lambda_{i}\left(\pi_{i}\right)$. Note that $\pi=\lambda_{i}\left(\pi_{i}\right)$ if and only if $\check{f}_{i}\left(t_{i}\right)=\check{f}(t)$, where $t_{i}=t_{i}\left(\pi_{i}\right), t=t(\pi)$, for all $f$ and $f_{i}=\tilde{\lambda}_{i}^{*}(f)$.

Note that $I_{0}(\mu)=I_{0}(\mu, 1), I_{1}(\mu)=I_{1}\left(\mu, \mu^{-1}\right)$ both lift (through $\left.\lambda_{0}, \lambda_{1}\right)$ to $I\left(\mu, 1, \mu^{-1}\right)$.

1.7. Integrals. There are several formal consequences concerning orbital integrals of functions $f, f_{i}$ related by $f_{i}=\tilde{\lambda}_{i}^{*}(f)$, since these integrals are the coefficients of $\check{f}$ and $\check{f}_{i}$. 
If $t_{1}=\left(t, t^{-1}\right)$ lies in $\widehat{T}_{1}$ then

$$
|T(R)| \sum_{\mathbf{m}=\left(m_{1}, m_{2}, m_{3}\right)} F_{f}(\mathbf{m}) t^{m_{1}-m_{3}}=\check{f}\left(\lambda_{1}\left(t_{1}\right)\right)=\check{f}_{1}\left(t_{1}\right)=\left|T_{1}(R)\right| \sum_{n} F_{f_{1}}(n) t^{n} .
$$

Comparing coefficients of $t^{n}$ we obtain

$$
\left|T_{1}(R)\right| F_{f_{1}}(n)=\sum_{\left\{\mathbf{m} ; m_{1}-m_{3}=n\right\}}|T(R)| F_{f}(\mathbf{m}) .
$$

A standard change of variables shows that this is the product of $\left|T^{\sigma}(R)\right|$, where $T^{\sigma}(F)=\{t$ in $T(F) ; t=\sigma(t)\}$, and

$$
\begin{aligned}
& F_{f}^{\sigma}(n)=\Delta(\gamma) \int f\left(g^{-1} \delta \sigma(g)\right) d g \\
& \qquad\left(\delta=(a, b, c), \quad \gamma=(a / c, c / a),|a / c|=|\boldsymbol{\pi}|^{n}\right) .
\end{aligned}
$$

It is clear that the integral on the right depends only on $n$, but not on the choice of $\delta$.

Remark. (1) Every $\check{f}_{1}$ is so obtained from some $\check{f}$, hence the $\check{f}_{1}$ separate the $\pi_{1}$. (2) we normalize the measures so that $\left|T_{i}(R)\right|=\left|T^{\sigma}(R)\right|$; the groups $T_{i}$ and $T^{\sigma}$ are isomorphic to the multiplicative group $\mathbf{G}_{m}$.

In the case of $H_{0}=\mathrm{SL}(2)$, taking a representative $t_{0}=(t, 1)$ in $\widehat{T}_{0}$ we have

$$
|T(R)| \sum_{\mathbf{m}} F_{f}(\mathbf{m}) t^{m_{1}-m_{3}}=\check{f}\left(\lambda_{0}\left(t_{0}\right)\right)=\check{f}_{0}\left(t_{0}\right)=\left|T_{0}(R)\right| \sum_{n} F_{f_{0}}(n) t^{n} .
$$

Hence $F_{f}^{\sigma}(n)=F_{f_{0}}(n)$, and the $\check{f}_{0}$ separate the $\pi_{0}$. In conclusion, we have

Lemma. If $\delta=(a, b, c), \gamma=(a / c, c / a)$, and $\gamma_{1}=(a, c)$, then $F_{f}^{\sigma}(\delta)=$ $F_{f_{0}}(\gamma)$ and $F_{f}^{\sigma}(\delta)=F_{f_{1}}\left(\gamma_{1}\right)$.

1.8. Norm. To extend the study of lifting from the unramified case to any admissible $\sigma$-invariant representation, we shall use the description of the stable $\sigma$-conjugacy classes and norm map $N$ from [I, §1]. Here and in (1.9), $F$ is any local field. Recall that two elements $\delta, \delta^{\prime}$ of $G(F)$ are called (stably) $\sigma$-conjugate if there is $h$ in $G(F)$ (resp. $G(\bar{F})$ ) with $\delta^{\prime}=h \delta \sigma\left(h^{-1}\right)$. The map $\delta \rightarrow \delta \sigma(\delta)$ induces a bijection $N$ from the set of stable $\sigma$-conjugacy classes in $G(F)$ to the set of stable conjugacy classes in $H(F)$. The norm map has a particularly simple description in the case where $\delta \sigma(\delta)$ has distinct eigenvalues. Up to a $\sigma$-conjugacy such $\delta$ can be assumed to be of the form $\delta=(a e)_{1}$ where

$$
a=\left(\begin{array}{ll}
a & b \\
c & d
\end{array}\right), \quad e=\left(\begin{array}{cc}
-1 & 0 \\
0 & 1
\end{array}\right), \quad a_{1}=\left(\begin{array}{lll}
a & 0 & b \\
0 & 1 & 0 \\
c & 0 & e
\end{array}\right)
$$

Then $\gamma=N \delta=(-1 / \operatorname{det} a) a^{2}$.

1.9. Weyl integration formula. Let $\left\{T_{0}\right\}$ denote a set of representatives for the conjugacy classes of Cartan subgroups of $H$ over $F$. The Jacobian of the morphism $T_{0} \times T_{0} \backslash H \rightarrow H,(t, g) \rightarrow g^{-1} t g$, is

$$
D(t)=|\operatorname{det}(1-\operatorname{Ad} t)|_{\mathrm{Lie} H_{t} \backslash H} \mid
$$


(see $[I, \S 2])$, and we have the Weyl integration formula

$$
\int f_{0}(g) d g=\sum_{\left\{T_{0}\right\}}\left|W\left(T_{0}\right)\right|^{-1} \int_{T_{0}(F)}|\Delta(t)|^{2} d t \int_{T_{0}(F) \backslash G(F)} f_{0}\left(g^{-1} t g\right) \frac{d g}{d t},
$$

where $W\left(T_{0}\right)$ is the Weyl group of $T_{0}$, and $\left|\Delta(t)^{2}\right|=|D(t)|$.

The analogue of this formula in the twisted case is based on the observation of (1.8) that each elements in $G(F)$ with regular norm is $\sigma$-conjugate to an element $\delta=(a e)_{1}$ with $a$ in $\operatorname{GL}(2, F)$. Note [I] that $\delta=(a e)_{1}$ and $\delta^{\prime}=$ $\left(a^{\prime} e\right)_{1}$ are $\sigma$-conjugate if and only if $a^{\prime}=(1 / \operatorname{det} b) b^{-1} a b$, so that we may take the $a$ in $N Z(K) \backslash T(F)$, where $T$ ranges over a set of representatives for the conjugacy classes of Cartan subgroups of $\mathrm{GL}(2)$ over $F, K$ is the splitting field of $T$ over $F, Z$ is the center of $\mathrm{GL}(2)$ and $N$ is the norm form $K$ to $F$. The Jacobian of the morphism $T \times G_{\delta}^{\sigma} \backslash G \rightarrow G$ by $(a, g) \rightarrow g^{-1} \delta \sigma(g)$ is $|\operatorname{det}[1-\operatorname{Ad} \delta \times \sigma]|_{\text {Lie } G_{\delta}^{\sigma} \backslash G} \mid$, which is equal to $|2| \Delta(\gamma)^{2}$, where $\gamma=N \delta=$ $(-1 / \operatorname{det} a) a^{2}$. The twisted Weyl integration formula is then

$$
\int f(g) d g=\frac{1}{2} \sum_{\{T\}} \int_{N Z(K) \backslash T(F)}|2| \Delta(\gamma)^{2} \omega_{\delta} \int_{G_{\delta}^{\sigma}(F) \backslash G(F)} f\left(g^{-1} \delta \sigma(g)\right) \frac{\omega_{G}}{\omega_{\delta}} .
$$

The Weyl group of $T(F)$ in $\operatorname{GL}(2, F)$ consists of two elements.

\section{LOCAL LIFTING}

2.1. Characters. Throughout this section, we let $F$ be a local (archimedean or not) field, $f_{i}$ a compactly supported smooth function on $H_{i}(F), \pi_{i}$ an admissible irreducible representation of $H_{i}(F)$, and $\pi_{i}\left(f_{i}\right)$ the convolution operator $\int f_{i}(g) \pi_{i}(g) d g$; implicit is a choice of a Haar measure. This operator has finite rank. A well-known result of Harish-Chandra (see [H, Theorem 5], when $F$ is nonarchimedean) asserts that there exists a locally-integrable complex-valued conjugacy-class function $\chi_{i}=\chi_{\pi_{i}}$ on $H_{i}(F)$, which is smooth on the regular set and called the character of $\pi$, such that

$$
\operatorname{tr} \pi_{i}\left(f_{i}\right)=\int f_{i}(g) \chi_{i}(g) d g
$$

for all $f_{i}$.

The twisted analogue of $[\mathrm{H}$, Theorem 5], (see [Cl]), asserts that given a $\sigma$ invariant admissible irreducible representation $\pi$ of $G(F)$, there exists a $\sigma$ conjugacy class function $\chi$ on $G(F)$ with the above properties, such that

$$
\operatorname{tr} \pi(f \times \sigma)=\int f(g) \chi(g) d g
$$

for all $f$. The trace class operator $\pi(f \times \sigma)$ is defined in (1.2). Note that $\chi$ is the twisted character of $\pi$; it is not the character in the usual sense.

We need the character and its properties for the orthogonality relations of (3.3), as well as for the study of the approximation in [IV, §1], and lifting in [IV, §2].

2.2. Packets. The local lifting is defined (in (2.3)) by means of character identities, and we need to relate characters of packets, not individual characters. The packet of a representation of $G(F)$ or $H_{1}(F)$ will consist of that representation alone. In the case of $H(F)=\operatorname{SL}(2, F)$, packets are defined in [LL], 
and we repeat here the definition. A representation $\pi$ of $H(F)$ is contained in the restriction of an irreducible representation $\tilde{\pi}$ of $\mathrm{GL}(2, F)$ to $H(F)$. The set of irreducible constituents in the restriction of $\tilde{\pi}$ to $H(F)$ is called the packet of $\pi$. This set depends on $\pi$ alone, and is denoted by $\{\pi\}$. It consists of a single element, unless $\tilde{\pi} \otimes \chi \simeq \tilde{\pi}$, where $\chi$ is a nontrivial character of $F^{\times}$, necessarily of order 2 . In the latter case $\{\pi\}$ consists of two or four elements. Moreover, $\chi$ determines a quadratic extension $K$ of $F$, and $\tilde{\pi}$ is associated to a two-dimensional representation of the Weil group $W_{F}$ of $F$ induced from a character $\theta$ of the Weil group $W_{K}$ of $K$. This $W_{K}$ is of index two in $W_{F}$. Then $\tilde{\pi}$ is denoted by $\tilde{\pi}(\theta)$; it is in the discrete series if $\theta \neq \bar{\theta}$, where $\bar{\theta}(z)=\theta(\bar{z})$ and the bar denotes the action of $\operatorname{Gal}(K / F)$. Now $\{\pi\}$ consists of two elements if $\tilde{\pi}=\tilde{\pi}(\theta)$ and $z \rightarrow \theta(z / \bar{z})$ is not of order (one or) two $\left(z\right.$ in $\left.K^{\times} \simeq W_{K / K}=W_{K} / W_{K}^{c}\right)$, and of four elements if $\theta(z / \bar{z})$ is of order two. The elements in the packet $\{\pi\}$ are the orbit of $\pi$ under the action $\pi \rightarrow \pi^{g}$ of $\left(g\right.$ in) $\mathrm{GL}(2, F)$, where $\pi^{g}(h)=\pi\left(g^{-1} h g\right)$. We denote by $\chi_{\{\pi\}}$ the sum of $\chi_{\pi^{\prime}}$, where $\pi^{\prime}$ ranges over $\{\pi\}$. Then $\gamma \rightarrow \chi_{\pi}(\gamma)$ is a class function, namely $\chi_{\pi}\left(g^{-1} \gamma g\right)=\chi_{\pi}(\gamma)$ for $g$ in $H(F)$. The character $\chi_{\{\pi\}}$ is a stable class function, namely $\chi_{\{\pi\}}\left(g^{-1} \gamma g\right)=\chi_{\{\pi\}}(\gamma)$ for all $g$ in GL $(2, F)$, or $\chi_{\{\pi\}}(\gamma)=\chi_{\{\pi\}}\left(\gamma^{\prime}\right)$ whenever $\gamma, \gamma^{\prime}$ are stably conjugate elements in $H(F)$ $\left(\gamma, \gamma^{\prime}\right.$ have the same eigenvalues). Note that the character is defined only on the regular set.

2.3. Lifting. We can now turn to the definition of local lifting.

Definition. The representation $\pi_{0}$ of $H_{0}(F)$ lifts to the representation $\pi$ of $G(F)$ if $\chi_{\pi}(\delta)=\chi_{\left\{\pi_{0}\right\}}(\gamma)$ whenever $\gamma=N \delta$ is a regular element of $H(F)$. In this case we write $\pi=\lambda_{0}\left(\pi_{0}\right)$.

Remark. This definition is based on the definition of the norm $N$ in [I], which is recalled in (1.8). The norm relates stable $\sigma$-conjugacy classes in $G(F)$ and stable conjugacy classes in $H(F)$. It was noted in $(2.2)$ that $\chi_{\{\pi\}}(\gamma)$ is a stable class function. To be a lift of $\pi_{0}$ the (twisted) character $\chi_{\pi}$ of $\pi$ has to be a stable $\sigma$-class function, namely $\chi_{\pi}(\delta)=\chi_{\pi}\left(\delta^{\prime}\right)$ if $\delta$ and $\delta^{\prime}$ are stably $\sigma$-conjugate.

2.4. Lemma. $\pi=\lambda_{0}\left(\pi_{0}\right)$ if and only if $\operatorname{tr} \pi(f \times \sigma)=\operatorname{tr}\left\{\pi_{0}\right\}\left(f_{0}\right)$ for all $f, f_{0}$ with $f_{0}=\lambda_{0}^{*}(f)$.

Remark. As in [I, §3], we write $f_{i}=\lambda_{i}^{*}(f)$ for functions with matching orbital integrals.

Proof. Suppose that $\operatorname{tr} \pi(f \times \sigma)=\operatorname{tr}\left\{\pi_{0}\right\}\left(f_{0}\right)$. We use the Weyl integration formula of (1.9) to write $\operatorname{tr} \pi(f \times \sigma)=\int f(g) \chi(g) d g$ as

$$
|2| \sum_{\{T\}} \frac{1}{2} \int_{N Z(K) \backslash T(F)} \Delta(\gamma)^{2} \chi_{\pi}\left((a e)_{1}\right) \Phi_{f}^{\sigma}\left((a e)_{1}\right) \omega_{\delta} .
$$

Fix a quadratic extension $K$ of $F$. Denote by $T_{K}(F)$ the element of $\{T\}$ (i.e. torus in $\operatorname{GL}(2, F))$ which splits over $K$. Take $f$ so that its twisted orbital integral $\Phi_{f}^{\sigma}$ is supported on $T_{K}(F)$, namely on the $\sigma$-orbits of the $\delta=(a e)_{1}$ with $a$ in $T_{K}(F)$. We claim that

$$
\operatorname{tr} \pi(f \times \sigma)=\frac{|2|}{2} \int_{Z(F) \backslash T_{K}(F)} \Delta(\gamma)^{2} \chi_{\pi}(\delta) \Phi_{f}^{\text {st }}(\delta) \omega_{\delta} \quad\left(\delta=(a e)_{1}\right),
$$


where $\Phi_{f}^{\text {st }}(\delta)$ denotes the stable twisted orbital integral of $f$ at $\delta$, as in [I, $\left.\S 3\right]$. To show this, note that the trace $\operatorname{tr} \pi(f \times \sigma)$ depends only on the stable twisted orbital integral $\Phi_{f}^{\sigma}$ of $f$, since it is equal to $\operatorname{tr}\left\{\pi_{0}\right\}\left(f_{0}\right)$. If we take $f_{0}=0$, then for each $a$ in $T_{K}(F)$ we have

$$
\Phi_{f}^{\sigma}\left((u a e)_{1}\right)=-\Phi_{f}^{\sigma}\left((a e)_{1}\right) \quad\left(u \text { in } F-N_{K / F} K\right) .
$$

Since $\operatorname{tr} \pi(f \times \sigma)$ vanishes for such $f$, we have

$$
\int_{Z(F) \backslash T_{K}(F)} \Delta(\gamma)^{2}\left[\chi_{\pi}\left((a e)_{1}\right)-\chi_{\pi}\left((u a e)_{1}\right)\right] \Phi_{f}^{\sigma}\left((a e)_{1}\right) \omega_{\delta}=0 .
$$

Choosing $f$ so that the support of $\Phi_{f}^{\sigma}\left((a e)_{1}\right)$ is small, we deduce that

$$
\chi_{\pi}\left((a e)_{1}\right)=\chi_{\pi}\left((\text { uae })_{1}\right)
$$

depends only on the stable $\sigma$-conjugacy class of $(a e)_{1}$. Hence, the claim follows.

On the other hand,

$$
\begin{aligned}
\operatorname{tr}\left\{\pi_{0}\right\}\left(f_{0}\right) & =\int f_{0}(g) \chi_{\left\{\pi_{0}\right\}}(g) d g \\
& =\sum_{\left\{T_{0}\right\}}\left[W\left(T_{0}\right)\right]^{-1} \int_{T_{0}(F)} \Delta(\gamma)^{2} \chi_{\left\{\pi_{0}\right\}}(\gamma) \Phi_{f_{0}}(\gamma) \omega_{\gamma} \\
& =\frac{1}{2} \int_{T_{0 K}(F)} \Delta(\gamma)^{2} \chi_{\left\{\pi_{0}\right\}}(\gamma) \Phi_{f_{0}}^{\text {st }}(\gamma) \omega_{\gamma} .
\end{aligned}
$$

The last equality follows from our assumption on $f_{0}$ that the stable orbital integral $\Phi_{f_{0}}^{\text {st }}(\gamma)$ of $f_{0}$ at $\gamma$ is supported on (the stable conjugacy class of) the torus $T_{0 K}(F)$ in $\left\{T_{0}\right\}$ which splits over $K$. Since the map $F^{\times} \backslash K^{\times} \rightarrow K^{1}$ by $z \rightarrow z / \bar{z}$ is a bijection and serves to relate measures from $Z(F) \backslash T_{K}(F)$ to the torus $T_{0 K}(F)$ of $\operatorname{SL}(2, F)$, and $f_{0}=\lambda_{0}^{*}(f)$ means $\Phi_{f}^{\text {st }}(\delta)=\Phi_{f_{0}}^{\text {st }}(\gamma)$ for all $\delta, \gamma$ with $N \delta=\gamma$, it follows that $\pi=\lambda_{0}\left(\pi_{0}\right)$. Note [I, Lemma 2.3.1] that the measures are related by $2 \omega_{\delta}=\omega_{\gamma}$.

2.5. Remark. The opposite direction is proven by reversing the above steps.

2.6. Recall that the norm map $N_{1}$ of [I] bijects the stable $\sigma$-conjugacy classes in $G(F)$ with the (stable) conjugacy classes in $H_{1}(F)=\mathrm{SO}(3, F)$. Recall that in each stable $\sigma$-conjugacy class of elements $\delta$ such that $\delta \sigma(\delta)$ has distinct eigenvalues, there are two $\sigma$-conjugacy classes (unless the eigenvalues of $\delta \sigma(\delta)$ lie in $F^{\times}$when there is a single $\sigma$-conjugacy class), and they differ by whether $G_{\delta^{\prime}}^{\sigma}(F)$ is split or not for a representative $\delta$. Here we put $\delta^{\prime}=\frac{1}{2}\left(\delta+J^{t} \delta J\right)$ as in [I, §1], and note that the $\sigma$-centralizer $G_{\delta^{\prime}}^{\sigma}$ of $\delta^{\prime}$ depends only on the $\sigma$-conjugacy class of $\delta$, up to conjugacy in $G(F)$.

The twisted character $\chi_{\pi}$ is a $\sigma$-class function on the $\sigma$-regular set, namely, $\chi_{\pi}\left(g^{-1} \delta \sigma(g)\right)=\chi_{\pi}(\delta)$ for all $g$ in $G(F)$. By an unstable $\sigma$-class function we mean a $\sigma$-class function which satisfies $\chi_{\pi}(\delta)=-\chi_{\pi}(\tilde{\delta})$ whenever $\delta, \tilde{\delta}$ are stably $\sigma$-conjugate but not $\sigma$-conjugate.

Note that if $\tilde{\delta}, \delta$ are stably $\sigma$-conjugate, but not conjugate, then up to $\sigma$ conjugacy $\delta=(a e)_{1}$ and $\tilde{\delta}=(u a e)_{1}$ with $u$ in $F^{\times}$but not in $N_{K / F} K^{\times}$, where $K / F$ is a quadratic separable extension determined by $\delta$. 
Definition. The representation $\pi_{1}$ of $H_{1}(F)$ lifts to the representation $\pi$ of $G(F)$ if $\chi_{\pi}$ is an unstable $\sigma$-class function and

$$
\left|\left(1+\gamma^{\prime}\right)\left(1+\gamma^{\prime \prime}\right)\right|^{1 / 2} \chi_{\pi}(\delta)=\chi_{\pi_{1}}\left(\gamma_{1}\right)
$$

for all $\gamma_{1}$ in $H_{1}(F)$ and $\delta$ in $G(F)$ such that $G_{\delta^{\prime}}^{\sigma}(F)$ is split and $N_{1} \delta=\gamma_{1}$ has distinct eigenvalues as an element of $H_{1}(F)=\mathrm{SO}(3, F)$. Here $\gamma^{\prime}, \gamma^{\prime \prime}$ denote the eigenvalues of $\gamma_{1}$ distinct from 1 . Note that $\chi_{\pi}(\delta)=-\chi_{\pi}\left(\delta^{\prime}\right)$ whenever $\delta$, $\delta^{\prime}$ are stably $\sigma$-conjugate but not $\sigma$-conjugate. We then write $\pi=\lambda_{1}\left(\pi_{1}\right)$.

2.7. Lemma. We have $\operatorname{tr} \pi(f \times \sigma)=\operatorname{tr} \pi_{1}\left(f_{1}\right)$ for all $f, f_{1}$ with $f_{1}=\lambda_{1}^{*}(f)$ if and only if $\pi=\lambda_{1}\left(\pi_{1}\right)$.

Remark. As in [I, §3], we write $f_{1}=\lambda_{1}^{*}(f)$ for functions $f_{1}, f$ on $H_{1}(F)$, $H(F)$ with matching orbital integrals, namely when

$$
\Phi_{f_{1}}\left(\gamma_{1}\right)=\left|\left(1+\gamma^{\prime}\right)\left(1+\gamma^{\prime \prime}\right)\right|^{1 / 2} \Phi_{f}^{l a b}(\delta)
$$

for $\delta, \gamma_{1}, \gamma^{\prime}, \gamma^{\prime \prime}$ as in (2.6). Here $\Phi_{f}^{l a b}(\delta)$ is the unstable twisted orbital integral of $f$ at $\delta$ (see [I, (3.5)]).

Proof. If $\operatorname{tr} \pi(f \times \sigma)=\operatorname{tr} \pi_{1}\left(f_{1}\right)$ for $f, f_{1}$ with $f_{1}=\lambda_{1}^{*}(f)$, then $\operatorname{tr} \pi(f \times \sigma)$ is equal to $\int f_{1}(g) \chi_{\pi_{1}}(g) d g$, which by the integration formula of (1.9), is

$$
\begin{aligned}
\sum_{\left\{T_{1}\right\}} & \frac{1}{2} \int_{T_{1}(F)} \Delta\left(\gamma_{1}\right)^{2} \chi_{\pi_{1}}\left(\gamma_{1}\right) \Phi_{f_{1}}\left(\gamma_{1}\right) d \gamma_{1} \\
\quad & =\sum_{\left\{T_{1}\right\}} \frac{1}{2} \int_{T_{1}(F)} \Delta\left(\gamma_{1}\right)^{2} \chi_{\pi_{1}}\left(\gamma_{1}\right)\left|\left(1+\gamma^{\prime}\right)\left(1+\gamma^{\prime \prime}\right)\right|^{1 / 2} \Phi_{f}^{l a b}(\delta) d \gamma_{1} .
\end{aligned}
$$

The sum is taken over a set of representatives for the conjugacy classes of Cartan subgroups $T_{1}$ of $H_{1}$ over $F$. Recall that $H_{1}=\mathrm{SO}(3) \simeq \operatorname{PGL}(2)$, and in $H_{1}$ a stable conjugacy class is a conjugacy class. The element $\delta$, or rather its $\sigma$ conjugacy class, is uniquely determined by $\gamma_{1}$ and the requirement that $G_{\delta^{\prime}}^{\sigma}$ be split over $F$. Moreover, $\Phi_{f}^{l a b}(\tilde{\delta})$ is $-\Phi_{f}^{l a b}(\delta)$ if $\delta, \tilde{\delta}$ are stably $\sigma$-conjugate but not $\sigma$-conjugate.

Define $\chi_{\pi}$ by the equation (2.6.1) to be an unstable $\sigma$-conjugacy class function. Then our sum becomes

$$
|2| \sum_{\{T\}} \frac{1}{2} \int_{Z(F) \backslash T(F)} \Delta(\gamma)^{2} \chi_{\pi}(\delta) \Phi_{f}^{l a b}(\delta) \omega_{\delta} .
$$

The sum is over conjugacy classes of $F$-tori in $\operatorname{GL}(2, F), \delta=(a e)_{1}, \gamma=$ $(-1 / \operatorname{det} a) a^{2}$, and $a \rightarrow \gamma_{1}$ defines an isomorphism of $Z(F) \backslash T(F)$ and $T_{1}(F)$ for tori $T, T_{1}$ which share their splitting field. Note that when the eigenvalues of $a e$ are $u, v$, then those of $\gamma$ are $u / v, v / u$, we have

$$
\Delta(\gamma)=\left|\left(1-(u / v)^{2}\right)\left(1-(v / u)^{2}\right)\right|^{1 / 2}
$$

and

The sum is equal to

$$
\Delta\left(\gamma_{1}\right)=|(1-u / v)(1-v / u)|^{1 / 2} .
$$

$$
|2| \sum_{\{T\}} \frac{1}{2} \int_{N Z(K) \backslash T(F)} \Delta(\gamma)^{2} \chi_{\pi}(\delta) \Phi_{f}^{\sigma}(\delta) \omega_{\delta} .
$$


This is

$$
\int f(g) \chi_{\pi}(g) d g
$$

by the twisted Weyl formula (1.9). Hence $\pi=\lambda_{1}\left(\pi_{1}\right)$ by the definition of $\chi_{\pi}$.

2.8. Induced. As in (1.3) let $\pi=I(\eta)$ denote the representation of $G(F)$ (normalizedly) induced from the character $\eta(a, b, c)=\mu(a / c)$ of the Borel subgroup $B(F)$, where $\mu$ is a character of $F^{\times}$. Denote by $\pi_{0}=I_{0}(\mu)$ and $\pi_{1}=I_{1}(\mu)$ the representations of $H_{0}(F), H_{1}(F)$ induced from the characters $\left(\begin{array}{ll}a & 0 \\ 0 & a^{-1}\end{array}\right) \rightarrow \mu(a),\left(\begin{array}{ll}a & 0 \\ 0 & b\end{array}\right) \rightarrow \mu(a / b)$ of the corresponding upper triangular Borel subgroups. Then the computation of (1.4) and the integration formulae of (1.9) show that $\pi=I(\eta)$ has a character $\chi$ which vanishes at $\delta$ unless $\delta$ is diagonal (up to $\sigma$-conjugacy), where

$$
\chi_{\pi}(\delta)=\Delta(\gamma)^{-1}(\eta(\delta)+\eta(\tilde{\delta})) \quad(\tilde{\delta}=J \delta J) .
$$

Similar standard computations show that $\chi_{\pi_{i}}$ are also supported on the (conjugacy classes of) diagonal elements of $H_{i}(F)$. They are given there by

$$
\chi_{\pi_{0}}(\gamma)=\Delta(\gamma)^{-1}\left(\mu(a)+\mu\left(a^{-1}\right)\right), \quad \gamma=\left(\begin{array}{cc}
a & 0 \\
0 & a^{-1}
\end{array}\right)
$$

and

$$
\chi_{\pi_{1}}\left(\gamma_{1}\right)=\Delta\left(\gamma_{1}\right)^{-1}\left(\mu(a)+\mu\left(a^{-1}\right)\right), \quad \gamma_{1}=\left(\begin{array}{ll}
a & 0 \\
0 & 1
\end{array}\right) .
$$

It follows that if $\pi=I(\eta), \pi_{0}=I_{0}(\mu), \pi_{1}=I_{1}(\mu)$, then

2.8.1. Lemma. $\pi=\lambda_{0}\left(\pi_{0}\right)=\lambda_{1}\left(\pi_{1}\right)$, namely $I_{0}(\mu)$ and $I_{1}(\mu)$ both lift to $I(\eta)$.

Proof. The character of $\pi, \pi_{i}$ are supported on the split tori, and the stable $\sigma$-conjugacy class of an element where $\chi_{\pi}$ does not vanish consists of a single $\sigma$-conjugacy class:

Remark. Here the field $F$ is any (archimedean or not) local field.

2.9. Special representation. Let $\nu$ denote the valuation character of $F^{\times}$, thus $\nu(x)=|x|$. The composition series of the induced representation $I_{0}=I_{0}(\nu)$ of $H(F)$ consists of the one-dimensional representation $\pi_{0}$ of $H(F)$ and the special representation $\mathrm{sp}$ of $H(F)$. Note that $\mathrm{sp}$ is irreducible if $F \neq \mathbf{R}$. But by (2.8.1) $I_{0}$ lifts to the representation $\pi=I(\eta)$ of $G(F)$, induced from the character $\eta=\left(\nu, 1, \nu^{-1}\right)$ of the upper triangular Borel subgroup of $G(F)$. The composition series of $\pi$ consists of the trivial representation $\pi(\eta)$, the irreducible representation $\pi_{P_{1}}\left(\operatorname{sp}(\nu, 1), \nu^{-1}\right)$ normalizedly induced from the representation $\operatorname{sp}(\nu, 1) \times \nu^{-1}$ of the maximal parabolic subgroup $P_{1}$ of type $(2,1)$, and the reducible representation $I_{P_{2}}\left(\nu, \operatorname{sp}\left(1, \nu^{-1}\right)\right)$ induced from the maximal parabolic $P_{2}$ of type $(1,2)$. This last representation has composition series consisting of the irreducible $\pi_{P_{2}}\left(\nu, \operatorname{sp}\left(1, \nu^{-1}\right)\right)$ and the Steinberg representation St. This result is due to Bernstein-Zelevinsky [BZ] when $F$ is nonarchimedean. Now $I_{P_{2}}\left(\nu, \operatorname{sp}\left(1, \nu^{-1}\right)\right)$ is not $\sigma$-invariant, but $\mathrm{St}$, being the unique square-integrable irreducible constituent of $I(\eta) \simeq{ }^{\sigma} I(\eta)$, is $\sigma$-invariant. Hence, $\pi_{P_{2}}\left(\nu, \operatorname{sp}\left(1, \nu^{-1}\right)\right)$, as well as $\pi_{P_{1}}\left(\operatorname{sp}(\nu, 1), \nu^{-1}\right)$ (for the same reason), is not $\sigma$-invariant. The one-dimensional representation $\pi(\eta)$ of $G(F)$ is clearly $\sigma$-invariant. Hence, $\operatorname{tr} I(\eta)(f \times \sigma)=\operatorname{tr} \operatorname{St}(f \times \sigma)+\operatorname{tr} \pi(\eta)(f \times \sigma)$. 
2.9.1. Lemma. The trivial (resp. special) representation of $H(F)$ lifts to the trivial (resp. Steinberg) representation of $G(F)$.

Proof. As the characters of both $\pi_{0}$ and $\pi(\eta)$ are identically one, and the packets of the trivial and of the special representations of $H(F)$ consists of a single element each, the lemma follows at once from the definition (2.3) of the lifting.

Remark. The only $\sigma$-invariant one-dimensional representation $\pi$ of $G(F)$ is the trivial one. Indeed, $\pi$ is given by a character $\beta$ of $F^{\times}$(namely, $\pi(g)=$ $\beta(\operatorname{det} g)$ ) of order 3, thus $\beta^{3}=1$. But $\pi$ is $\sigma$-invariant only if $\beta=\beta^{-1}$. Hence $\beta=1$ and $\pi$ is trivial, as asserted.

\section{ORTHOgONALITY}

3.1. Orthogonality relations. The packets of irreducible admissible representations of $H(F)$ are described in (2.2). Their characters satisfy the orthogonality relations which we now recall.

For any stable conjugacy class functions $\chi, \chi^{\prime}$ on $H(F)$ put

$$
\left\langle\chi, \chi^{\prime}\right\rangle_{e}=\frac{1}{2} \sum_{\left\{T_{0}\right\}}\left[D\left(T_{0}\right)\right]\left|T_{0}(F)\right|^{-1} \int_{T_{0}(F)} \chi(\gamma) \bar{\chi}^{\prime}(\gamma) \Delta(\gamma)^{2} \omega_{\gamma} .
$$

The sum is taken over a set of representatives $T_{0}$ for the stable conjugacy classes of elliptic tori of $H$ over $F$. $\left[D\left(T_{0}\right)\right]$ is the number of conjugacy classes within the stable conjugacy class of $T_{0}$; it is 2 if $T_{0}$ is elliptic, 1 if $T_{0}$ is split. As usual, $\left|T_{0}(F)\right|$ denotes the volume of $T_{0}(F)$.

3.2. Lemma. Let $\pi$ and $\pi^{\prime}$ be admissible irreducible tempered representations of $H(F)$, at least one of which is square-integrable. Then $\left\langle\chi_{\{\pi\}}, \chi_{\left\{\pi^{\prime}\right\}}\right\rangle_{e}$ is 0 unless the packets $\{\pi\},\left\{\pi^{\prime}\right\}$ are equal, when it is equal to the number of irreducible constituents in $\{\pi\}$.

Proof. This follows from the orthonormality relations for characters of squareintegrable $H(F)$-modules (see [K, Theorem $\mathrm{K}$ ], for a general $p$-adic group).

3.3. Twisted orthogonality. Let $\pi$ be a $\sigma$-invariant supercuspidal representation of $G(F)$. Such $\pi$ do not exist unless the residual characteristic of $F$ is 2. (This is well known, and proven in [IV] using the trace formula.) As in (1.2) there is an intertwining operator $A$ from the space of $\pi$ to itself such that ${ }^{\sigma} \pi(g)=\pi(\sigma(g))=A \pi(g) A^{-1}$. Since $\pi$ is irreducible and $A^{2}$ intertwines $\pi$ with itself it is a scalar, which we may normalize to be 1 . Extend $\pi$ to a representation $\pi^{\prime}$ of $G^{\prime}(F)=G(F) \rtimes\langle\sigma\rangle$ by setting $\pi(\sigma)=A$. As noted in (2.1), the character $\chi_{\pi^{\prime}}$ of $\pi^{\prime}$ exists as a locally integrable function on $G^{\prime}(F)$ which is smooth on the subset of $G(F) \times \sigma$ which consists of $\delta \times \sigma$ with regular $\gamma=N \delta$. If $G_{\delta}^{\sigma} \simeq H_{\gamma}$ is a compact torus of $H=\operatorname{SL}(2)$ over $F$ then the proof of [JL, Lemma 7.4.1], shows that

$$
\begin{aligned}
\chi_{\pi^{\prime}}(\delta \times \sigma)= & d\left(\pi^{\prime}\right) \int_{G(F)}\left[\left(\pi^{\prime}\left((g \times 1)^{-1} \cdot \delta \times \sigma \cdot g \times 1\right) u, \tilde{u}\right)\right. \\
& \left.+\left(\pi^{\prime}\left((g \times \sigma)^{-1} \cdot \delta \times \sigma \cdot g \times \sigma\right) u, \tilde{u}\right)\right] \omega_{G} \\
= & 2 d\left(\pi^{\prime}\right)\left|G_{\delta}^{\sigma}(F)\right| \int_{G_{\delta}^{G}(F) \backslash G(F)}\left(\pi^{\prime}\left(g^{-1} \delta \sigma(g) \times \sigma\right) u, \tilde{u}\right) \frac{\omega_{g}}{\omega_{\delta}} .
\end{aligned}
$$


Here $d\left(\pi^{\prime}\right)$ denotes the formal degree of $\pi^{\prime} ; u, \tilde{u}$ are vectors in the space of $\pi^{\prime}$ and the contragredient of $\pi^{\prime}$, with $(u, \tilde{u})=1$.

The orthogonality relations for characters imply the following.

3.3.1. Lemma. Let $\pi_{2}$ be a unitary $\sigma$-invariant irreducible admissible represention of $G(F)$ and $\pi$ a $\sigma$-invariant supercuspidal representation of $G(F)$. Suppose that the function $\delta \rightarrow \chi_{\pi^{\prime}}(\delta \times \sigma)$ is a stable $\sigma$-conjugacy class function on $G(F)$. Define $\left\langle\chi_{\pi^{\prime}}, \chi_{\pi_{2}^{\prime}}\right\rangle_{e}$ to be

$$
\frac{1}{2} \sum_{\{T\}}|Z(F) \backslash T(F)|^{-1} \int_{Z(F) \backslash T(F)} \Delta(\gamma)^{2} \chi_{\pi^{\prime}}(\delta \times \sigma) \sum_{\delta^{\prime}} \bar{\chi}_{\pi_{2}^{\prime}}\left(\delta^{\prime} \times \sigma\right) \omega_{\gamma} .
$$

It is equal to 0 unless $\pi$ and $\pi_{2}$ are equivalent when it is equal to 1 .

Remark. The sum over $T$ ranges over a set of representatives for the conjugacy classes of elliptic tori in $\mathrm{GL}(2)$ over $F$. The sum over $\delta^{\prime}$ ranges over a set of representatives for the $\sigma$-conjugacy classes within the stable $\sigma$-conjugacy class of $\delta$. For $a$ in $T(F)$ we have $\delta=(a e)_{1}$, and there are two $\delta^{\prime}$ in our case of $\delta$ with compact $G_{\delta}^{\sigma}(F) \simeq H_{\gamma}(F), \gamma=N \delta$.

Proof. First suppose that $\pi_{2}$ is equivalent to $\pi$. Put $\pi_{i}^{\prime}=\omega^{i} \otimes \pi^{\prime}(i=0,1)$, where $\omega$ is the character of $G^{\prime}(F)$ which attains the value 1 on $G(F)$ and the value -1 at $\sigma$. The representations $\pi_{0}^{\prime}, \pi_{1}^{\prime}$ are inequivalent. Put

$$
\bar{\phi}(g)=d\left(\pi^{\prime}\right)\left(\pi^{\prime}(g) u, \tilde{u}\right), \quad \pi_{1}^{\prime}(\phi)=\int_{G^{\prime}(F)} \phi(g) \pi_{1}^{\prime}(g) d g .
$$

By the Schur orthogonality relations for the square-integrable representations $\pi_{i}^{\prime}$ we have

$$
\operatorname{tr} \pi_{0}^{\prime}(\phi)=1, \quad \operatorname{tr} \pi_{1}^{\prime}(\phi)=0
$$

Then

$$
1=\operatorname{tr} \pi_{0}^{\prime}(\phi)-\operatorname{tr} \pi_{1}^{\prime}(\phi)=2 \int_{G(F)} \phi(g \times \sigma) \chi_{\pi^{\prime}}(g \times \sigma) \omega_{G}
$$

By the Weyl integration formula (1.9) this is equal to

$$
\begin{aligned}
2 \cdot \frac{|2|}{2} \sum_{\{T\}} \int_{N_{Z}(K) \backslash T(F)} \Delta(\gamma)^{2} \chi_{\pi^{\prime}}(\delta \times \sigma) \omega_{\delta} \int_{G_{\delta}^{\sigma}(F) \backslash G(F)} \phi\left(g^{-1} \delta \sigma(g)\right) \frac{\omega_{g}}{\omega_{\delta}} \\
\quad=2 \cdot \frac{|2|}{2} \sum_{\{T\}} \int_{Z(F) \backslash T(F)} \Delta(\gamma)^{2} \chi_{\pi^{\prime}}(\delta \times \sigma) \omega_{\delta} \sum_{\delta^{\prime}} \int_{G_{\delta^{\prime}}^{\sigma}(F) \backslash G(F)} \phi\left(g^{-1} \delta^{\prime} \sigma(g)\right) \frac{\omega_{g}}{\omega_{\delta}} .
\end{aligned}
$$

Harish-Chandra's "Selberg principle" [ $\mathrm{H}^{\prime}$, Theorem 29] implies the vanishing of the inner integral if $G_{\delta}^{\sigma} \simeq H_{\gamma}$ is a torus of $H$ which splits over $F$. Otherwise the comment preceding the lemma implies that we obtain

$$
\frac{1}{2} \sum_{\{T\}}\left|G_{\delta}^{\sigma}(F)\right|^{-1} \int_{H_{\gamma}(F)} \Delta(\gamma)^{2} \chi_{\pi^{\prime}}\left(\delta^{\prime} \times \sigma\right) \sum_{\delta^{\prime}} \bar{\chi}_{\pi^{\prime}}\left(\delta^{\prime} \times \sigma\right) \omega_{\gamma} .
$$

We used the isomo rphism $Z(F) \backslash T(F) \simeq G_{\delta}^{\sigma}(F) \simeq H_{\gamma}(F)$, and the relation $2 \omega_{\delta}=\omega_{\gamma}$ of measures on the groups $G_{\delta}^{\sigma}, H_{\gamma}$, from [I, Lemma 2.3.1].

It remains to deal with the case where $\pi$ and $\pi_{2}$ are inequivalent. But then $\left(\omega^{i} \otimes \pi_{2}^{\prime}\right)(\phi)=0$ for both $i$, and the lemma follows using the same argument. 
3.4. Reformulation. In the notations of (3.3), if $\chi_{\pi^{\prime}}$ is a stable $\sigma$-class function, we may define a stable class function $\chi$ on $H(F)$ by $\chi(\gamma)=\chi_{\pi^{\prime}}(\delta \times \sigma)$, where $\gamma=N \delta$. Lemma 3.3.1 implies that

$$
\frac{1}{2} \sum_{\left\{T_{0}\right\}_{s}}\left|T_{0}(F)\right|^{-1}\left[D\left(T_{0}\right)\right] \int_{T_{0}(F)} \Delta(\gamma)^{2}|\chi(\gamma)|^{2} \omega_{\gamma}
$$

is equal to 1 , where the sum is taken over the stable conjugacy classes of elliptic tori of $H$ over $F$, and $\left[D\left(T_{0}\right)\right]$ (see 3.1) is equal to 2 .

3.5. Lemma. In the notations of (3.4), we have that $\left\langle\chi_{\pi^{\prime}}, \chi_{\pi^{\prime}}\right\rangle_{e}$ is 1 if $\pi$ is the $\sigma$-invariant Steinberg representation.

Proof. This follows from (3.1) and (2.9.1). Note that the packet of the special representation of $H(F)$ consists of a single element, and the orthogonality relation (3.1) for it follows from the orthogonality relation for the trivial representation of the group of elements of reduced norm 1 in the quaternion division algebra, and the correspondence of [JL].

3.6. Finally we record a special case of a twisted analogue of $[\mathrm{K}$, Theorem $\mathrm{G}]$. The proof in the twisted case, for arbitrary reductive not necessarily connected $p$-adic group, follows closely that of $[\mathrm{K}]$, and will not be given here. Thus, let $\pi, \pi^{\prime}$ be $\sigma$-invariant, tempered representations with characters $\chi, \chi^{\prime}$. Each of $\pi, \pi^{\prime}$ defines a unique (up to association) parabolic subgroup and a squareintegrable representation $\rho, \rho^{\prime}$ of its Levi factor. Then $\pi, \pi^{\prime}$ are called relatives if $\rho$ is equivalent to $\rho^{\prime}$. Recall that we have the inner product

$$
\left\langle\chi, \chi^{\prime}\right\rangle_{e}=\sum_{T}|T(F)|^{-1} \int \sum_{\delta} \Delta \chi(\delta) \Delta \bar{\chi}(\delta) d \gamma .
$$

The first sum ranges over a set of representatives for the stable conjugacy classes of elliptic tori in $H(F)$. The integral is over $\gamma$ in $T(F)$. The inner sum is over a set of representatives for the $\sigma$-conjugacy classes with $N \delta=\gamma$.

3.7. Lemma $[\mathrm{K}]$. If $\pi, \pi^{\prime}$ are not relatives then $\left\langle\chi, \chi^{\prime}\right\rangle_{e}=0$.

The same result holds also when $F$ is the field of real numbers.

In our case of $G=\operatorname{PGL}(3)$, a $G$-module normalizedly induced from a tempered one is irreducible, and we need only the following special case of the lemma.

Corollary. If $\pi, \pi^{\prime}$ are inequivalent $\sigma$-invariant tempered G-modules, then $\left\langle\chi, \chi^{\prime}\right\rangle_{e}=0$.

The methods of $[\mathrm{K}]$ do not afford computing the value $\langle\chi, \chi\rangle_{e}$. But in the case of any $\left(\sigma\right.$-stable) supercuspidal $\pi$, we have $\langle\chi, \chi\rangle_{e}=1$ by (3.3.1). In the local lifting theorem of [IV] we list all $\sigma$-stable elliptic $\pi$, and compute $\langle\chi, \chi\rangle_{e} ;$ it is equal to the cardinality of the packet which lifts to $\pi$.

\section{REFERENCES}

[B] A. Borel, Automorphic L-functions, Proc. Sympos. Pure Math., vol. 33, part II, Amer. Math. Soc., Providence, R. I., 1979, pp. 27-63.

[BZ] J. Bernstein and A. Zelevinsky, Induced representations of reductive p-adic groups, Ann. Sci. École Norm. Sup. 10 (1977), 441-472. 
[Cl] L. Clozel, Characters of non-connected, reductive p-adic groups, Canad. J. Math. 39 (1987), 149-167.

[F] Y. Flicker, Rigidity for automorphic forms, J. Analyse Math. 49 (1987), 135-202.

[I] _ On the symmetric-square: Orbital integrals, Math. Ann. 279 (1987), 173-191.

[III] _ On the symmetric-square: Twisted trace formula, J. Funct. Anal. 98 (1991), 194-210.

[IV] - On the symmetric-square: Applications of a trace formula, Trans. Amer. Math. Soc. 330 (1992), 127-156.

[V] Y. Flicker and D. Kazhdan, On the symmetric-square: Unstable local transfer, Invent. Math. 91 (1988), 493-504.

[VI] - On the symmetric-square: Total global comparison, preprint.

[H] Harish-Chandra, Admissible invariant distributions on reductive p-adic groups, Queen's $\mathrm{Pa}$ pers in Pure and Appl. Math. 48 (1978), 281-346.

$\left[\mathrm{H}^{\prime}\right] \quad \ldots$, Harmonic analysis on reductive p-adic groups, Lecture Notes in Math., vol. 162, Springer-Verlag, Berlin and New York, 1970.

[JL] H. Jacquet and R. Langlands, Automorphic forms on GL(2), Lecture Notes in Math., vol. 114, Springer-Verlag, Berlin and New York, 1970.

[K] D. Kazhdan, Cuspidal geometry of p-adic groups, J. Analyse Math. 47 (1986), 1-36.

[LL] J.-P. Labesse and R. Langlands, L-indistinguishability for SL(2), Canad. J. Math. 31 (1979), 726-785.

Department of Mathematics, Ohio State University, Columbus, Ohio 43210-1174

E-mail address: flicker@function.maps.ohio-state.edu 